\title{
La educación inclusiva en la Educación Superior: relato del contexto universitario cubano
}

\author{
Elsie Alejandrina Pérez Serrano ${ }^{1}$
}

\section{Introducción}

La educación cubana de las últimas dos décadas evidencia un proceso sostenido de transformaciones que ha estado mediado por la influencia de dos factores esenciales: las nuevas tendencias mundiales entorno a la inclusión educativa y los cambios internos del país en relación con indicadores de naturaleza económica, política y social. De un modelo centrado en la escuela especial, como centro de recursos para los alumnos con necesidades educativas especiales, se transita a la constitución de un modelo educativo que busca respuesta a las necesidades de todos los alumnos, lo cual incluye el acceso, cada vez más creciente, a la educación superior.

Las bases ideológicas que sustentan el cambio derivan de las complejas relaciones de orden político, económico y axiológico que existen entre la educación y la sociedad. Ello presupone modificar la concepción de los procesos educativos para la inclusión, tanto en sus bases teóricas como en el orden de las políticas, para su implementación en la práctica. En el presente trabajo se explican los fundamentos epistemológicos y las políticas que favorecen la inclusión educativa en la Educación Superior cubana de los alumnos, cuyas necesidades educativas especiales se relacionan con diferencias en el desarrollo que demandan de recursos

1 UHo - Universidad de Holguín. Holguín - Cuba. Bolsista Capes/Brasil. eperez@fh.uho.edu.cu; elsieperezserrano@gmail.com 
no comunes. También se ilustra este acontecer con el ejemplo de la Universidad de Holguín, institución ubicada en la provincia de Holguín, al norte de la región oriental de la República de Cuba.

\section{Fundamentos teóricos}

El proceso de inclusión educativa en Cuba tiene su sustento epistemológico en la integración de postulados teóricos de disciplinas esenciales: filosofía, antropología, sociología, psicología y pedagogía, entre otras. Estos postulados sostienen la naturaleza social de los seres humanos y que su desarrollo acontece a través de la unión indisoluble de sus componentes cuantitativos y cualitativos, biológicos y sociales, afectivos y cognitivos. Así mismo, permiten reconocer la existencia de vínculos e interdependencia entre los fenómenos, todo lo cual fundamenta las enormes posibilidades de desarrollo que tienen las personas con necesidades educativas especiales, asociadas a algún déficit.

Las directrices más generales parten de la filosofía marxista-leninista que aporta la concepción materialista de la dialéctica y la teoría del reflejo, lo cual posibilita la comprensión del papel activo del hombre en la sociedad y en la creación de su propio destino. Otra de las categorías importantes que aporta es la de la actividad, la cual expresa la esencia genérica del hombre, su sustancia creadora y examina al sujeto de la actividad en el orden socio-histórico. Esta teoría defiende que la condición de hombre es de naturaleza social, que no es un factor que se sobrepone a lo biológico y, por tanto, las modificaciones biológicas no condicionan su desarrollo, elemento que integra la visión optimista de esta teoría.

La inclusión como perspectiva axiológica universal, con singularidad en la educación, requiere de un posicionamiento en el orden antropológico. Y es que cada tipo histórico concreto de sociedad, tiene su modelo de hombre, el cual se define a partir del modelo antropológico de cada época, o sea, la manera en que concibe y entiende la esencia del hombre, dentro de la cultura en que vive, en un grado determinado de su desarrollo. En la actualidad ese modelo de hombre es el de la diversidad, sin exclusiones por diferencias derivadas de las características personales o del ambiente cultural. A su vez, implica ponerse en el lugar del "otro" y desde su realidad comprenderle, lo que permite alternar la perspectiva propia con la ajena, o sea, avanzar en dirección de la alteridad.

En el campo sociológico, constituye una idea esencial la educación multicultural como fenómeno político y social de reivindicación de los derechos humanos y civiles de todas las personas, lo que promueve prácticas más inclusivas. También constituye una idea esencial la mirada que proporciona el concepto de la diversidad humana al significado social de las personas diferentes y las relaciones que se establecen entre estas ideas con el currículo y la reproducción cultural, lo que revaloriza el papel que tienen las influencias externas organizadas en el desarrollo del sujeto. 
La escuela de psicología histórico-cultural aporta postulados esenciales que fundamentan la raíz social de las funciones psíquicas superiores como resultado de la apropiación de la experiencia histórico-social. Todo lo cual sostiene el carácter activo y transformador de la psiquis humana y el papel de los instrumentos de naturaleza cultural en el proceso de desarrollo humano. Constituyen conceptos de referencia para el proceso de inclusión aquellos que notifican el origen social de las funciones psíquicas superiores del hombre, lo cual le concede una concepción optimista a su desenvolvimiento, como la ley genética de las funciones psíquicas superiores, la situación social del desarrollo y la zona de desarrollo próximo.

Desde la pedagogía, un referente general es el Ideario Martiano en relación con el papel de la instrucción y la educación para la formación del ser humano. De esta manera, se considera la existencia de una pedagogía única, que conceptualiza el proceso de enseñanza-aprendizaje, sus principales categorías, la formación de valores en los estudiantes y la comunicación educativa en un escenario común. A su vez, contempla la incorporación de procedimientos específicos y de adecuaciones curriculares, ajustadas a las demandas de los estudiantes, que posibiliten el alcance de los objetivos de formación.

Entre los fundamentos teóricos de la inclusión educativa se encuentran como referentes ineludibles un conjunto de documentos que han contribuido a interpretar de manera no excluyente la educación de las personas con algún déficit, los cuales se relacionan a continuación: el Informe Warnock (1978), el Programa de Acción Mundial para las Personas con Discapacidad (1981), The Standard Rulers (1994), la Conferencia Mundial sobre Necesidades Educativas: Acceso y Calidad, Marco de Acción (1994) y la Guía para la evaluación y mejora de la educación inclusiva (Index for Inclusion), de Booth y Ainscow (2002).

Por último, se erige como un fundamento importante la existencia de un marco legal, coherente con las posiciones de avanzadas, respecto al derecho de todos los ciudadanos a una educación estatal, gratuita y de calidad. A partir de los fundamentos referidos en apretada síntesis, a continuación se expone la concepción teórico-metodológica que configura el proceso de inclusión en las instituciones de la Educación Superior en la República de Cuba.

\section{Dimensiones y procesos de la inclusión educativa en la Educación Superior en Cuba}

Los postulados teóricos referidos poseen una función lógica-gnoseológica ya que ayudan a comprender el proceso de inclusión, de lo cual se deriva su función metodológica para explicarlo y establecer la vía a seguir. La concepción de la inclusión educativa en Cuba se estructura a partir de elementos que caracterizan dicho proceso y permiten abstraerlo y modelarlo. Particularmente se consideran dos componentes: las dimensiones y sus procesos, que se explicarán seguidamente. 
Las dimensiones determinan los elementos generales e integradores que representan el funcionamiento y la especificidad del proceso de inclusión e incluyen un conjunto de elementos específicos que constituyen sus indicadores. Se consideran tres dimensiones para el proceso de inclusión educativa en general y para la educación superior en particular: la axiológica, referida a la aceptación de la diversidad humana como un valor; la política, con la adopción de una legislación para concretar el derecho a la inclusión; la educativa, que implica la modelación didáctica del proceso de enseńanza-aprendizaje. A continuación se explican brevemente cada una de ellas y los principales indicadores que sustentan las acciones que se despliegan para garantizar la inclusión en este nivel de enseńanza.

La dimensión axiológica, a partir de la aceptación de la diversidad como norma del desarrollo humano, implica considerar el concepto de patrón de normalidad no en términos estadísticos, sino en la multiplicidad de las diferencias humanas desde uno a otro punto de su desarrollo. Ello presupone modificar el lenguaje como reflejo del cambio ideológico que también envuelve la eliminación de las etiquetas excluyentes, o sea, no se trata de la educación de personas ciegas, sordas o con capacidades físico-motoras limitadas, entre otros déficits que originan necesidades educativas especiales, sino que se trata de personas en cuya situación social de desarrollo se implican, entre múltiples factores, algunos como la ceguera, la sordera o la limitación para trasladarse.

Desde esta dimensión se favorece la plena participación e integración de los esfuerzos combinados de la familia, la comunidad, los servicios educativos, de la salud, la cultura, el deporte, además de los profesionales implicados en ellos y la asistencia social para ofrecer igualdad de oportunidades que favorezca la inclusión. Esta perspectiva proyecta modificar las actitudes hacia las personas diferentes por parte de la población, los docentes, los funcionarios de la educación, la familia y por ellos mismos, pues como afirmó Vigotsky (1995, p.26) refiriéndose al desarrollo, este "tiene sus propias leyes, su ritmo, sus ciclos, sus desproporciones, sus metamorfosis, su traslado de los centros y sus estructuras".

La dimensión política, establece el marco legal para la implementación de las medidas requeridas que transformen las prácticas. Implica especificar las responsabilidades de cada organismo e institución para que contribuya a articular políticas educativas favorables a los jóvenes con necesidades educativas especiales, así como las medidas de sanidad, bienestar social y trabajo que les permita conseguir una vida adulta independiente. Ello presupone la adopción de una legislación para cambiar la manera de entender la universidad y la adopción de estrategias para la sensibilización de los actores sociales que establezcan su participación y la de las instituciones, así como la toma de decisiones por las administraciones de las universidades.

Estas políticas incluyen la formación de recursos humanos, la organización de ellos y de los recursos materiales y técnicos, así como las normativas para el 
acceso de estas personas a la Educación Superior, su permanencia en las instituciones universitarias y el acompañamiento durante el egreso, con lo cual se cierra el ciclo de la inclusión en los niveles laboral y social. Las políticas públicas avistan normas generales en los documentos legales para garantizar el derecho a la inclusión y su concreción en la actividad de las instituciones. Pero no se limitan a eso sino que han de ser, ante todo, un marco de inspiración a los profesores y los directivos para la actividad investigativa en las universidades.

La dimensión educativa expresa los planteamientos en torno a la formación para desplegar al máximo las posibilidades del estudiante, teniendo en cuenta la unidad de influencias proveniente de los diferentes contextos. Esta dimensión emerge de las relaciones que se establecen entre diversos indicadores que se detallan posteriormente, de lo cual resulta como cualidad trascendental el desarrollo superior de cada estudiante. De esta manera la inclusión se interpreta no como un problema sino como una oportunidad para la mejora de las instituciones universitarias.

Desde esta dimensión se decide el éxito de la inclusión, donde no basta respetar y promover la democracia de las diferencias, en armonía con la heterogeneidad del alumnado y la contextualización del proceso referido, sino que se precisa de la organización de todos sus componente, a partir de la existencia de un currículo flexible que posibilite la realización de adecuaciones que estimulen las potencialidades de cada alumno. Ello presupone introducir alternativas, incitar la participación protagónica de los estudiantes, eliminar la crítica negativa y valorizar las opiniones individuales, con la incorporación de un sistema de evaluación que respete estos elementos y no altere el equilibrio emocional. A continuación, se presentan los principales indicadores de esta dimensión.

La gestión administrativa implica introducir transformaciones en las dinámicas de las universidades a partir de tres procesos esenciales: el acceso, la permanencia y el egreso, los cuales se explican con posterioridad. El marco legal contempla la implementación de estrategias de información sobre la legislación y las oportunidades derivadas de ella para acceder a la Educación Superior.

El establecimiento también precisa de procedimientos institucionales que garanticen la continuidad de los estudios de estos alumnos hasta su culminación y el seguimiento en los centros de trabajo de cada uno, con lo cual se cierra el ciclo de la inclusión social. Así mismo, incluye la toma de decisiones respecto a financiamientos para la eliminación de barreras arquitectónicas y la adquisición de tecnologías adecuadas para potenciar el aprendizaje de los alumnos.

La capacitación comprende la preparación del claustro para conducir el proceso de enseńanza-aprendizaje de estos alumnos. Ello se concreta en las variadas modalidades de la superación y el trabajo metodológico en los diferentes niveles organizativos. De manera que los profesores y los trabajadores de las universidades en general adquieran conocimientos sobre las características, demandas y poten- 
cialidades de estos estudiantes. Con ello se viabiliza la inclusión en los diferentes espacios y procesos sustantivos universitarios, entendiendo por tales la docencia, la investigación, la práctica profesional y la extensión universitaria.

Los profesores, además, requieren de ayudas para incorporar en su labor pedagógica recursos didácticos y tecnológicos que habitualmente les son desconocidos, como en el caso de un alumno con ceguera, la escritura Braille y programas de computación para invidentes. La capacitación abarca la formación de recursos humanos que no son frecuentes en estos espacios, como el intérprete de lengua de señas.

Los recursos técnicos trata de la adquisición de tecnologías y equipamientos necesarios que favorezcan el proceso de enseñanza-aprendizaje. En muchos casos implica la asesoría de especialistas para el uso de las tecnologías de la información y las comunicaciones, o la participación de profesionales de la educación especial que posibiliten su incorporación a la metodología de las clases.

La extensión comunitaria se dirige a dos direcciones; la primera es la incorporación del estudiante a las actividades que la universidad proyecta en las comunidades y, a su vez, movilizar a las instituciones de estos espacios a favorecer la participación de ellos. La otra dirección es garantizar la práctica en los centros laborales en correspondencia con las exigencias que la formación profesional requiere.

La modelación didáctica se relaciona con la incorporación de variados recursos didácticos, metodológicos y tecnológicos que no son habituales en el proceso de enseńanza-aprendizaje que se despliega en las universidades. Implica que los profesores deben estructurar de forma consciente y planificada el proceso de estimulación de las potencialidades de los estudiantes para satisfacer sus demandas educativas, lo cual presupone tres elementos básicos:

- La ayuda preferentemente en el componente de orientación de las tareas docentes y otras actividades.

- La mediación de las demás personas que incluye a los profesores, los demás estudiantes, entre otros.

- La óptima configuración del escenario educativo y social en sentido general.

Una vez concluido el análisis de las dimensiones resulta imprescindible abordar el componente de los procesos. Por estos se hace referencia a aquellos que permiten materializar en tiempo y espacio la inclusión en la Educación Superior, ofreciendo las mayores oportunidades a la diversidad del estudiantado. Como se enunció anteriormente estos procesos son: acceso, permanencia y egreso; su contenido conlleva al diseño de un sistema armónico de alternativas que posibiliten el empleo eficiente de los recursos humanos y materiales de que dispone cada centro.

El acceso tiene como contenido el establecimiento de las condiciones requeridas para garantizar el paso exitoso de los estudiantes con necesidades educativas especiales por el sistema de ingreso a las instituciones de la Educación Superior. Se cuenta con una normativa legal que establece políticas educativas denomina- 
da: Sobre el tratamiento a los jóvenes discapacitados para estudiar en la Educación Superior (CUBA, 2010).

La permanencia tiene como objetivo la formación integral y profesional de los estudiantes con necesidades educativas especiales, a través de los componentes laboral, académico e investigativo del plan de estudio correspondiente. La modelación de este proceso incluye el establecimiento de pronósticos que permitan potenciar el aprendizaje del alumno, teniendo en cuenta la articulación entre las aspiraciones y necesidades individuales con las colectivas.

El egreso trata del acompañamiento del recién graduado con necesidades educativas especiales a las instituciones de trabajo, en vínculo con la familia y los factores comunitarios. Está dirigido a incorporarle a un sistema de relaciones nuevas que son decisivas para la inclusión social, con lo cual se favorece su actuación profesional y la realización personal en correspondencia con las exigencias de la sociedad.

Un elemento decisivo para la inclusión de los alumnos en la Educación Superior es la estructura del currículo. En Cuba este es común para todas las instituciones de Educación Superior y se conforma de la forma siguiente: currículo base que es determinado por la Comisión Nacional de Carrera y es del 70\% al $80 \%$ del total de horas. El currículo propio que lo determina cada universidad y responde a necesidades de la región, el cual oscila entre un $10 \%$ y un $20 \%$. Y el currículo optativo y electivo que es determinado por los estudiantes, al cual corresponde el 10\% del total de horas del plan de estudio. Esta estructuración posibilita la realización de adecuaciones curriculares que permitan satisfacer las demandas específicas de los alumnos con necesidades educativas especiales.

\section{Normativas para el acceso a la Educación Superior en Cuba}

Las personas con necesidades educativas especiales, ya sean estudiantes o trabajadores, acceden a la universidad mediante el mismo proceso que el resto de los ciudadanos. No obstante, existen regulaciones complementarias que establecen condiciones y prioridades para contribuir a su acceso.

En Cuba el proceso de ingreso a la Educación Superior está dirigido por la Dirección de Ingreso del Ministerio de Educación Superior. A su vez, se crea una Comisión de Ingreso Provincial que tiene a la universidad de cada territorio como institución rectora y responsable por la legalidad del mismo. A su vez, incorpora a un conjunto de organismos e instituciones que tienen participación en los procesos educacionales.

El sistema de ingreso a la Educación Superior en Cuba establece los requisitos siguientes para todos los aspirantes:

- Tener la educación media aprobada que, en Cuba, significa concluir el $12^{\circ}$ grado o curso equivalente. Se requiere tener una nota mínima de 60 puntos. 
- Aprobar los exámenes de ingreso en las tres materias básicas: español, matemática e historia. Se requiere tener una nota mínima de 60 puntos.

Con estos resultados se elabora un escalafón a nivel de cada una de las 15 provincias que comprende la división político-administrativa del país y el municipio especial de Isla de la Juventud. Para la elaboración de dicho escalafón se establece el procedimiento siguiente: $50 \%$ de los puntos se obtiene de la media de las calificaciones de la educación media aprobada y el otro $50 \%$ es la media de los exámenes de ingreso, también aprobados. El estudiante puede seleccionar hasta 10 carreras universitarias y la plaza se concede a partir de las disponibilidades existentes, las solicitudes realizadas por él y en correspondencia con el número obtenido en el escalafón.

Las vías para acceder a la Educación Superior en Cuba son las siguientes:

- Los jóvenes, al concluir la educación media.

- Los trabajadores que alcanzan la educación media.

- Por concurso, al que tienen acceso los dos anteriores.

- Para desmovilizados del servicio militar (Orden 18 del Ministro de las FAR).

Para los alumnos con necesidades educativas especiales se tienen en cuenta otras condiciones que son normadas en la Circular MES-Mined del 2010, Sobre el tratamiento a los jóvenes discapacitados para estudiar en la educación superior (CUBA, 2010, p.2). Este documento en sus diferentes por cuantos, reglamenta un conjunto de elementos que se sintetizan a continuación:

- Ellos no compiten con el resto de los estudiantes por una plaza.

- Para acceder a la Educación Superior solo tienen que cumplir con los dos requisitos ya expuestos: tener aprobada la educación media y realizar las pruebas de ingreso, obteniendo 60 puntos o más en cada una.

- Alcanzan la carrera solicitada sin entrar en el escalafón que se conforma en cada provincia, es decir, con independencia de la calificación obtenida. Ellos solo compiten con otros alumnos que tienen condiciones similares y que opten por la misma carrera solicitada.

- Los que se presentan por la modalidad de concurso reciben el mismo tratamiento al que se hace referencia anteriormente, y se les concede la carrera sin afectar las plazas destinadas a los demás solicitantes.

En el acto de realización de las pruebas de ingreso se les brinda un tratamiento especial que incluye un conjunto de ayudas, entre las que se encuentran las siguientes:

- La disposición de un local individual si lo requieren y con las condiciones de acceso.

- Los alumnos sordos o hipoacúsicos son acompañados con un intérprete de lengua de señas y se excluyen del dictado en la prueba de español. 
- Los alumnos con baja visión cuentan con el acompañamiento de un profesor para transcribir sus respuestas si es necesario.

- Los alumnos con baja visión son eximidos de la pregunta de geometría en la prueba de matemática.

- La extensión del tiempo de examen, si las características del alumno lo requirieran. El tiempo máximo establecido para cada examen es de 4 horas, sin embargo, ellos utilizan las que demanden.

El documento precisa que las instituciones universitarias pueden establecer otras medidas e incluir los apoyos que se consideren necesarios para cada alumno en particular, como: materiales a relieve o grabados, ordenador y maquina Perkin, entre otros. También, deja a la decisión de cada provincia la incorporación de maestros de la educación especial para la calificación de los exámenes que se realicen en Braille o para alumnos con déficits motores complejos.

Las pruebas de ingreso a la Educación Superior se realizan entre los meses de mayo y julio de cada año, mediante dos convocatorias que brinda mayores oportunidades a los que no aprueban en la primera. La Circular MES-Mined del 2010, ya referida, establece que en el mes de febrero, las direcciones provinciales de la Enseñanza Especial en coordinación con las asociaciones: Asociación Nacional de Sordos de Cuba, Asociación Nacional de Ciegos y la Asociación Cubana de Limitados Físico Motores enviarán a la Dirección de Ingreso del Ministerio de Educación Superior, el listado de los alumnos que aspiran a estudios superiores. Esta Circular en su artículo $8^{\circ}$ declara que puede ser ratificada o renovada todos los ańos, según las características específicas de cada curso escolar.

Las carreras con mayores resultados en los procesos inclusivos en Cuba son las siguientes: Derecho, Informática, Ciencias Técnicas, Fisioterapia, Educación, Educación Especial y Educación Física. Tradicionalmente, la carrera que primero dio acceso a estos estudiantes es la de Derecho, aun cuando no se hablaba de integración ni de inclusión. Estos datos son coherentes con los consultados por las estadísticas de otros países.

Los estudiantes con necesidades educativas especiales que en mayor medida acceden a la Educación Superior en Cuba se corresponden con los déficits que se relacionan a continuación y, siguiendo un orden de frecuencia, ellos son: la ceguera y baja visión, las limitaciones físico-motoras, los trastornos en el lenguaje, la sordera e hipoacusia y algunos trastornos mentales. En la consulta bibliográfica se comprueba la coincidencia con los referidos por diferentes países.

\section{Un ejemplo, la Universidad de Holguín}

La provincia de Holguín se encuentra ubicada al norte de la región oriental, se divide en 11 municipios y cuenta con una población que supera el millón de habitantes, de 11 millones que tiene el país en su totalidad. La Universidad de Holguín 
"Oscar Lucero Moya" tiene 41 años de fundada. En la actualidad tiene una matrícula de 5483 alumnos en la graduación y de ellos el 56,6\% son mujeres.

La Universidad tiene alumnos con necesidades educativas especiales por diferentes causas como: ceguera, sordera o hipoacusia, limitaciones en el desarrollo físico-motor, trastornos en el lenguaje y con dificultades mentales que apenas llega al 1\% del total de la matrícula. O sea, es bajo el número de estudiantes con demandas específicas en el aprendizaje que continúan su formación en la Educación Superior, lo cual acontece de forma similar en universidades de Cuba y otros países. A continuación se ilustra con ejemplos la actividad que la Universidad despliega a favor de la inclusión educativa.

Las acciones que ejemplifican el trabajo que despliega la Universidad se referencian, de la participación de la autora en una mesa redonda realizada, en el marco del VI Congreso Brasileiro de Educación Especial (2014).

Ejemplos de acciones para la dimensión axiológica:

- Divulgación de la vida de personas con necesidades educativas especiales que han concluido estudios universitarios, incluyendo aquellos que en la actualidad son trabajadores de la institución.

- Espacio cinematográfico del Núcleo de Estudios de Género de la Universidad y el Instituto Cubano de Cine "Del silencio al grito", con actividades a favor del respeto a la diversidad e incluye a las personas que tienen necesidades educativas especiales.

- Debates con los profesores sobre temas como: planos y niveles de la diversidad y el cambio de la visión de las limitaciones y las deficiencias de los alumnos hacia sus competencias y capacidades.

- Participación sistemática de los profesores-investigadores a programas de la televisión para contribuir a la modificación de actitudes respecto a la diversidad humana.

- Apoyo en el tránsito de la Universidad hacia la institución laboral, respecto a la sensibilización del colectivo al cual se insertan y la aceptación para contribuir a estimular sus potencialidades.

Ejemplos de acciones para la dimensión política:

- Compra y entrega gratuita por la universidad de ordenadores personales a los alumnos con ceguera.

- Eliminación de barreras arquitectónicas, de comunicación y de participación.

- Seguimiento durante dos años después del egreso de la Universidad para proveer las ayudas que sean necesarias en las instituciones de trabajo. 
- Tramitación de convenio de trabajo con la Dirección Provincial de Educación para incorporar las escuelas especiales del territorio a la labor con estos alumnos, en aspectos como:

- la utilización de sus recursos cuando los alumnos de la universidad lo necesiten y el servicio de los talleres especializados como la elaboración de materiales en Braille;

- la colaboración de los directivos, profesores y especialistas de la escuela especial para el proceso de ingreso a la Educación Superior, la capacitación del claustro y la participación en investigaciones, entre otras.

Ejemplos de acciones para la dimensión educativa:

- Adecuaciones al plan de estudio y de acceso al currículo, con la incorporación de disciplinas que se requieran e incremento del tiempo de estudio según las necesidades del alumno.

- Establecimiento del diseño de adecuación curricular en la Educación Superior que incluye elementos como: determinación del equipo de trabajo, apoyos (directos, indirectos y complementarios) y estrategias didácticas, entre otros que resulten necesarios para cada estudiante.

- Incorporación de estrategias de colaboración en grupo con los alumnos de mejor nivel de preparación.

- Designación de tutores de apoyo seleccionados del claustro de la carrera o de estudiantes de carreras pedagógicas como parte de su práctica docente.

- Responsabilizar con la atención al colectivo de año en el aula ordinaria con el jefe de año como profesor coordinador.

- Garantía de los medios materiales necesarios para el uso del sistema de comunicación alternativo Braille como: máquina Perkins, el programa de computación para invidentes, Jaws, los libros y diccionarios hablados, y los medios a relieve

- Evaluación del aprendizaje con un abordaje más cualitativo, integrador y enfocado en el desempeño, y otras adecuaciones como la realización de un año preparatorio, promover con una evaluación inferior a la establecida en algunas disciplinas.

- Articulación de ambientes educacionales: universidad, hogar, instituciones educacionales y sociales, tales como Joven Club de Computación, bibliotecas y escuelas especiales.

- Participación en las actividades de la extensión universitaria, mediante la realización de acciones que permitan la articulación entre las universidades, las organizaciones no gubernamentales, la comunidad y la familia en actividades deportivas, culturales y la práctica laboral. 
- Conversatorios y conferencias con el grupo de estudiantes y el colectivo de profesores por especialistas del territorio.

- Incorporación de clases de consulta individual por las diferentes disciplinas.

- Garantía de apoyo psicológico, educacional y médico.

- Otorgamiento de beca en la Universidad a un familiar cuando precisan de ayuda para el validismo, con alimentación y alojamiento gratuito.

Los procesos de inclusión en la Universidad no se circunscriben a los alumnos que estudian las diferentes carreras para su graduación. Ellos, también, han tenido acceso a la educación de postgrado, donde han cursado especializaciones y maestrías. En la actualidad se inicia la formación doctoral que ya es una realidad en otras universidades del país. Así mismo, la inclusión ha alcanzado al colectivo de profesores y de los trabajadores en general pues la universidad cuenta entre sus recursos humanos personas que tienen algún déficit en el desarrollo. Estos profesores posen alta calificación, incluyendo titulación de doctorado.

A continuación se ilustra con dos ejemplos el proceso de inclusión en la Universidad de Holguín, uno de graduación y otro que tiene concluido pos-graduación. El primer ejemplo, es un alumno con ceguera y el otro ejemplo, es una alumna con dificultades en el desarrollo físico-motor.

Alumno C. A. G., de sexo masculino y oriundo de una zona rural en la cual realiza la educación básica y media. Padece de retinosis pigmentaria, una enfermedad ocular crónica de origen genético, con degeneración progresiva de la retina y pérdida de sus células principales: conos y bastones. Él presentó una diminución lenta y progresiva de la agudeza visual en ambos ojos, primero de la visión nocturna y el campo visual periférico, después de la visión central, lo cual conlleva a la realización de cirugía a los 17 años. Esta se realiza en período cercano a los exámenes de fin de curso y no realiza el reposo visual orientado, surgiendo la ceguera.

La ausencia de visión y la carencia de preparación para autovalerse en una escuela de régimen interno, le impiden continuar la educación media por lo cual, después de recibir un curso de alfabetización en Braille, logra concluirla en la modalidad para adultos de espacios rurales. Se presenta a las pruebas de ingreso por concurso, las que aprueba, y solicita la carrera de Licenciatura en Inglés como segunda lengua, la cual le es concedida.

Esta carrera es de requisitos adicionales que conllevan la realización de pruebas de aptitudes y tiene un ańo preparatorio el cual se debe aprobar con nota mínima de 4 puntos. $^{2} \mathrm{Al}$ iniciar el curso con la realización del diagnóstico pedagógico, se concluye que tiene baja competencia en lengua materna, pocas aptitudes para la lengua inglesa, con características personales de timidez y baja aceptación de la ayuda que se ofrece para la solución de las tareas, además nunca

2 La escala de calificaciones para la Universidad en Cuba es de 2 a 5 puntos y el aprobado es 3 puntos. 
recibió cursos de inglés. El colectivo de año le sugiere cambio de carrera, pero él y su mamá deciden que no.

La estrategia pedagógica que se elabora incluye acciones individuales para la gestión del proceso de enseñanza-aprendizaje y con la adecuación curricular correspondiente. Como parte de las acciones, se incorpora un año de preparación para trabajar las habilidades comunicativas para la lengua materna e inglesa. Se ubica un profesor de apoyo del claustro de la carrera, licenciado en inglés, que empezó a recibir preparación en Braille por parte de la escuela especial.

Se incluye la realización de un ciclo de conversatorios y conferencias por especialistas de la propia universidad y la directora de la escuela especial, con el grupo de estudiantes y el colectivo de profesores. Todos los estudiantes del grupo asumieron tareas específicas y por disciplinas para ayudarle.

La escuela especial se comprometió con la elaboración de los materiales en Braille y a relieve que fuesen necesarios, para lo cual se vinculan estudiantes de años superiores, quienes también programaron actividades de estudio por materias. Se le facilita una máquina Perkins, papel, presillas, grabadora y computador personal. En el currículo se incluyen dos materias a desarrollar por profesores de la escuela especial: Movilidad y Estenografía. También se brinda apoyo psicológico, transporte para las consultas médicas y beca para él y su mamá con alimentación y alojamiento gratuito.

A pesar del trabajo realizado por el colectivo de año y la carrera, además de las adecuaciones al plan de estudio y de acceso al currículo, al concluir los dos cursos preparatorios, este alumno no alcanza los objetivos de las disciplinas y, nuevamente, se le sugiere el cambio de carrera. Decide estudiar Derecho y ya tiene culminado el primer año con buenos resultados académicos.

Alumna S. L. C., de sexo femenino, de la ciudad de Holguín, a los 8 años padece una enfermedad del sistema nervioso del tipo de las neuropatías, que le afecta el sistema motor, quedando con parálisis en las extremidades inferiores y rigidez en las superiores, así como la necesidad de una silla de ruedas para desplazarse. Actualmente tiene 27 años y es Vice-presidente de la Asociación de limitados físicos motores (Aclifim) en la provincia de Holguín, está casada y tiene una hija de 6 años.

Se matricula en la carrera de Sociología en curso para trabajadores, donde se realizan adecuaciones complementarias, o sea, se le proporcionan los apoyos para la realización de tareas básicas en el orden material y tecnológico. Concluye los estudios con excelentes resultados en el orden académico y la extensión universitaria, promoviendo la actividad deportiva en la comunidad, favoreciendo la inclusión de las personas que se desplazan en silla de rueda.

$\mathrm{Al}$ año de concluir la universidad se presenta al proceso de selección del Programa de Maestría en Ciencias Sociales y Axiología. Tiene artículos publicados en 
Cuba y España y ha dirigido proyectos socioculturales para la inclusión social, entre sus resultados científicos se encuentran los siguientes:

- La discapacidad no constituye diferencias entre el mundo y yo (2006).

- La maternidad: ¿sueño o realidad de la mujer discapacitada cubana? (2007).

- Experiencias de la provincia Holguín en la integración de las personas con discapacidad al arte (2010).

- Influencia de los tabúes para la inclusión social en la diversidad funcional (personas con discapacidad) (2014).

Defiende su tesis de maestría en el 2014, con el título: La inclusión social de las personas con diversidad funcional física desde una concepción axiológica para favorecer el desarrollo humano. En la actualidad inicia los estudios de doctorado con el tema "Las representaciones sociales de las personas con diversidad funcional en el contexto de la Educación Superior".

\section{Conclusiones}

La Educación Superior en Cuba muestra avances en los procesos de inclusión de las personas con necesidades educativas especiales, que se configura a través de las dimensiones: axiológica, política y educativa, las cuales reflejan la complejidad del objeto. La inclusión educativa en este nivel de enseńanza requiere de acciones concretas en los procesos de acceso, permanencia y egreso, expresando una articulación entre su contenido y el de las dimensiones referidas.

No existe un modelo de inclusión único para los alumnos con necesidades educativas especiales en la Educación Superior, lo cual depende de las particularidades de cada país y de las demandas específicas de cada estudiante. En Cuba, la educación es un derecho básico, por lo tanto, el gobierno establece las oportunidades para que, desde un contexto único y normalizador, se garantice la Educación Superior a estos alumnos.

Las universidades cubanas cuentan con un marco legal que les permite diseñar acciones para adoptar la opción más potenciadora del desarrollo de cada uno de sus alumnos. En ellas se despliegan proyectos de investigación que incluyen la búsqueda de recursos para el perfeccionamiento de las adecuaciones al plan de estudio y de acceso al currículo que posibiliten promocionar al máximo los desarrollos diferentes y singulares de los alumnos con necesidades educativas especiales.

\section{Referencias}

BOOTH, T.; AINSCOW, M. Index for Inclusion. Bristol: Center for Studies in Inclusive Education, 2002. 
CUBA. Ministerio de Educación. Resolución Ministerial n. 141/2001. La Habana, 2001.

CUBA. Circular Mined-MES. Sobre el tratamiento a los jóvenes discapacitados para estudiar en la Educación Superior/2010. La Habana, 2010.

SERRANO, E. P. Inclusão no Ensino Superior: das políticas públicas aos desafios institucionais. Mesa Redonda 8. VI Congresso Brasileiro de Educação Especial, São Carlos, 2014.

VYGOTSKY, L. S. Obras completas. Tomo V: Fundamentos de Defectología. La Habana: Pueblo y Educación, 1995. 336p.

\section{Resumo}

\section{A educação inclusiva na educação superior: relato do contexto universitário cubano}

Os principais fundamentos teóricos da inclusão no ensino superior em Cuba permitem modelar dois componentes essenciais: as dimensóes e os processos. As dimensóes configuram a inclusão em três perspectivas: a axiológica, referida à aceitação da diversidade humana como um valor; a política, com a adoção de uma legislação para concretizar o direito à inclusão e; a educativa, que implica a elaboraçáo de estratégias didáticas colaborativas entre os professores, os alunos e todas as pessoas que participam do contexto universitário. A inclusão impóe desafios às instituiçóes do ensino superior que implica introduzir transformaçóes em suas dinâmicas a partir de três processos: o aceso, a permanência e o egresso. Isso pressupóe a formação de recursos humanos, a formação permanente dos professores, a organização dos recursos materiais, humanos e técnicos e o trabalho didático metodológico no processo de ensino e de aprendizagem. Também, refere-se às chamadas "categorias de deficiências" com maior inclusão, os programas mais inclusivos e as adequaçóes curriculares que se realizam. Por último, o artigo apresenta um exemplo do estado atual da inclusão na Universidade de Holguín, com referência à graduação e pós-graduação.

Palavras-chave: Ensino Inclusivo. Ensino Superior. Necessidades Educativas Especiais. Universidade Cubana.

\section{Abstract \\ Inclusive Education in Higher Education: Cuban University Context Report}

The main theoretical foundations supporting inclusive education in Higher Education in Cuba allow the implementation of two essential components: its dimensions and processes. The dimensions comprise three perspectives: the axiological, based on the acceptance of human diversity as a value; the political, with development of a legislation to make possible the right to inclusion, and the educational, which involves the development of collaborative learning strategies among teachers, students and everybody else involved in the university context. The inclusion imposes challenges to the Higher Education institutions and implies the introduction of changes in their dynamics based on three processes: entrance, retention and graduation. This predicates the training of human resources, improvement of teachers' professional development, organization of material, human and technical resources as well as the methodological didactic work in the teaching-learning process. It also refers to the so called "categories of deficiencies" where most inclusive are blind people and with physical disabilities. The most inclusive university course is Law. Finally, it exemplifies the current state of the University of Holguin, referring to the education of undergraduates and postgraduates.

Keywords: Inclusive Education. Higher Education. Special Education Needs. Cuban University. 